\title{
Noradrenergic modulation of cognitive function: clinical implications of anatomical, electrophysiological and behavioural studies in animal models ${ }^{1}$
}

The early demonstration of the neurotransmitter role of norepinephrine (NE) within the brain stimulated intense study of its functions over the subsequent three decades. A large majority of brain noradrenergic neurons are concentrated in the brainstem nucleus, locus coeruleus (LC). This nucleus gives rise to an extensive and regionally specialized noradrenergic innervation of the CNS, including providing the sole source of $\mathrm{NE}$ to hippocampus and neocortex. Despite the intense examination of the LC/noradrenergic system, and the knowledge gained concerning various properties of this system, its functions remain enigmatic.

This editorial will provide a brief review of electrophysiological and behavioural studies which indicate that NE enhances cortical information processing through a variety of actions distributed across multiple anatomical regions and involving multiple receptors. It is proposed that the $\mathrm{LC}$ /noradrenergic system acts to create dynamic, widespread patterns of electrophysiological activity in neocortex and other forebrain areas that provide essential substrates for the operation of attentional and other cognitive processes. A greater understanding of the actions of the $\mathrm{LC} /$ noradrenergic system may provide the opportunity to treat cognitive dysfunction better in certain patient populations.

\section{CHARACTERISTICS OF THE LC/NORADRENERGIC SYSTEM}

The LC is a well-delineated cluster of noradrenergic neurons, located adjacent to the fourth ventricle in the pontine brainstem. It is composed of a small number of neurons (approximately 1600 per nucleus in rat, several thousand in monkey, and 10000-15000 in human). However, these cells possess immensely ramified axons such that the nucleus projects throughout the neuraxis, from spinal cord to neocortex (reviewed in Foote et al. 1983).

In rodents, there appears to be little topographic specificity in these projections in the sense that individual LC neurons project to widely dispersed regions. However, within terminal fields noradrenergic axons are characterized by regionally specific patterns of innervation. For example, LC axons preferentially innervate particular cortical regions and laminae, especially in primates (reviewed in Foote \& Morrison, 1987). The dense innervation of layer I is particularly interesting, given that this is considered an important site for cortical integration (Vogt, 1991). Heterogeneity is also observed at the receptor level. Thus, traditionally three noradrenergic receptor subtypes have been recognized: $\alpha_{1}, \alpha_{2}$, and $\beta$. $\alpha_{1}$ - and $\beta$-receptors are thought to exist primarily at post-synaptic sites whereas $\alpha_{2}$-receptors are believed to be present both pre- and post-synaptically; presynaptic $\alpha_{2}-$ receptors exert an inhibitory influence on LC neuronal discharge and NE release (Svensson et al. 1975), whereas the actions of post-synaptic $\alpha_{2}$-receptors are largely unknown. The distribution across and within cortical regions varies between the specific receptor subtypes, as does secondmessenger coupling. For example, $\beta$-receptors appear to be more broadly distributed across cortical laminae and are positively coupled to the Gs/cAMP second-messenger system, whereas $\alpha_{1}$ - and $\alpha_{2}$ receptors are concentrated in the superficial layers and are coupled to the phosphoinositol and

\footnotetext{
1 Address for correspondence: Dr C. W. Berridge, Department of Psychiatry, School of Medicine, University of California, San Diego, 9500 Gilman Drive, La Jolla, California 92093-0603, USA.
} 
Gi/cAMP systems, respectively (Dohlman et al. 1991). Recently, molecular biological studies have revealed an even greater diversity of adrenergic receptor subtypes indicating additional degrees of complexity (Jones \& Palacios, 1991). The distinct cellular functions mediated by the recently identified receptor subtypes remain largely unknown.

To summarize, the LC constitutes a system in which alterations in activity of a very small number of neurons can be broadcast to vast brain regions and neuronal populations of immense number. However, within this system there exists a degree of specificity conferred by the pattern of fibre termination, receptor subtype distribution, and second-messenger coupling that provides a potential substrate for the performance of multiple functions.

These anatomical characteristics suggest possible global actions of the LC/noradrenergic system, such as modulation of behavioural state. This hypothesis is buttressed by recordings from LC neurons in unanaesthetized animals. The pioneering work of Hobson \& McCarley and their colleagues demonstrated that these neurons exhibit their highest discharge rates during waking, evince very slow rates during slow-wave sleep, and are silent during REM or paradoxical sleep (Hobson et al. 1975). Furthermore, changes in their discharge rates anticipate changes in behavioural state (Hobson et al. 1975; Foote et al. 1980; Aston-Jones \& Bloom, 1981). Subsequent work, by several laboratories, has confirmed and extended these observations. Of particular interest in the present context is the observation that within the waking state, LC neurons discharge most rapidly in anticipation of EEG and behavioural changes that signal enhanced arousal or attentiveness (Foote et al. 1980; Aston-Jones \& Bloom, 1981). This suggests that enhanced LC activity, which presumably initiates enhanced NE release in forebrain and other target areas, may help to create widespread alterations in forebrain activity patterns that are reflected in EEG measures and are an integral part of epochs of enhanced alertness and particular cognitive functions.

This view is compatible with numerous studies demonstrating a facilitatory effect of NE on information processing by individual cortical and hippocampal neurons. To summarize briefly a vast literature, these studies show that the application of NE to such neurons can reduce spontaneous or weakly driven activity while at the same time preserving or enhancing responses to other, potent, specific synaptic inputs (Foote et al. 1975; Woodward et al. 1979). Thus activation of LC neurons might induce a state in which forebrain circuits are tuned to provide the greatest possible discrimination between optimal and non-optimal inputs. Clearly, such a scheme suggests an important role for the LC/NE system in attentional and other cognitive processes.

In addition to the ability of NE to enhance signal-to-noise ratios in target regions, a number of electrophysiological actions of the LC/NE system have recently been identified that support a modulatory role of the this system in global, state-dependent cognitive processes. These observations are briefly reviewed below.

\section{FOREBRAIN EEG}

The observation that changes in LC neuronal discharge rates precede changes in EEG state implies but does not demonstrate a causal role for the LC in EEG state modulation. To address this hypothesis better, we recently conducted a series of studies in which electrophysiological recordings were used to guide placement of an infusion needle in close proximity to the LC through which drugs that alter LC neuronal discharge rates were infused (Berridge \& Foote, 1991). Electrophysiological recordings verified and quantified the effects of these infusions on LC neuronal activity. It was observed that the selective enhancement of $\mathrm{LC}$ neuronal discharge resulted in a robust activation of forebrain EEG in anaesthetized rats. This EEG response was blocked by pretreatment with the $\beta$-noradrenergic antagonist, propranolol. Conversely, bilateral inhibition of LC neuronal discharge activity decreased signs of forebrain EEG activation (Berridge et al. 1991, 1993). In both the LC activation and inactivation studies, the onset and recovery of the EEG responses closely followed the changes in LC neuronal activity levels. Furthermore, localization studies indicated that infusion-induced changes in EEG state were not observed when infusions were placed 
outside the immediate vicinity of the LC. These observations indicate that, under these experimental conditions, the LC is a potent modulator of forebrain EEG state, with LC neuronal activity being causally related to the maintenance of EEG activity patterns associated with arousal.

These results are consistent with in vitro observations of $\mathrm{McC}$ Cormick and colleagues that $\mathrm{NE}$ enhances neuronal responsivity and induces a shift in firing pattern of cortical and thalamic neurons to a single-spike firing mode that is associated with arousal and/or attention (see McCormick et al. 1991). These effects are due to a complicated array of actions of NE at $\alpha_{1}$ - and $\beta$-receptors located on numerous cell types. The concerted actions of NE in both cortex and thalamus create a state in which the signal processing ability of forebrain neural systems is enhanced.

\section{ERPs/P300}

The responsiveness of $\mathrm{LC}$ neurons to stimuli that elicit an orientating response suggests the involvement of these neurons in attention-related cognitive processes. In humans, attention is often studied using event-related potentials (ERPs). ERPs are voltage fluctuations time-locked to sensory, motor, or cognitive events that are extracted from EEG recordings using signal averaging techniques. The P300 component of human ERPs is elicited in response to novel and/or task-related stimuli and is thus thought to have particular relevance to attentional or mnemonic processes.

In monkeys, P300-like components are also observed and have been studied in animal models of attention. The LC/NE system appears to be critical for the generation of a normal P300 response. Thus, bilateral LC lesions in squirrel monkeys selectively decreased P300 components of the ERP (Pineda et al. 1989). Similarly, inhibition of LC firing and NE release with systemically administered clonidine also decreases P300 components (Swick et al. 1988). These results suggest an important role of the $\mathrm{LC} /$ noradrenergic system in the modulation of cortical responsiveness to sensory information.

\section{LTP}

Potent modulatory actions of NE have been observed in an extensively studied model of cellular mechanisms of memory, long-term potentiation (LTP). LTP refers to a use-dependent, long-lasting increase in synaptic strength or efficacy: when excitatory synapses are rapidly and repetitively stimulated for brief periods (tetanic stimulation), the post-synaptic neurons generate action potentials more readily upon subsequent stimulation. In the hippocampal formation, three forms of LTP have been described; one involving mossy-fibre input to CA3 of the hippocampus (from the dentate gyrus), one involving the Schaffer collateral input to region CAl (from the entorhinal cortex), and one involving perforant path input to the dentate gyrus. The observation of LTP in a structure critical for memory function further motivates interest in LTP as a possible mechanism underlying memory.

NE has been demonstrated to influence LTP observed in CA3 and dentate gyrus. Thus, depletion of NE substantially decreases the population spike observed in dentate gyrus (Stanton \& Sarvey, 1985), whereas NE application elicits a frequency-dependent enhancement of LTP in the CA3 subfield (Hopkins \& Johnston, 1984). These effects appear to be dependent on actions of NE at $\beta$ receptors (Hopkins \& Johnston, 1988).

NE also elicits a long-lasting enhancement of synaptic efficacy in both the dentate gyrus and CA1 region of the hippocampus in vitro in the absence of tetanic stimulation. Thus, NE increases the population spike evoked by perforant path stimulation in the dentate gyrus (Stanton \& Sarvey, 1987) and by Schaffer collateral stimulation in the hippocampal CA1 region (Heginbotham \& Dunwiddie, 1991). The latter effects were attributable to actions of $\mathrm{NE}$ at $\beta$-receptors. In vivo, similar effects were observed in the dentate gyrus following either LC activation (Harley \& Sara, 1992) or NE application (Stanton \& Sarvey, 1987). These observations indicate a potentially critical role of the LC/noradrenergic system in mediating long-lasting modifications in synaptic efficacy. 


\section{BEHAVIOURAL STUDIES}

The above described observations indicate that NE enhances electrophysiological responses of individual neurons and neuronal ensembles, and can induce activity patterns associated with enhanced cortical signal processing throughout the forebrain. Based on these observations, it is posited that one function of the LC/noradrenergic system is the modulation of behavioural state and/or state-dependent processes. The ability of NE to enhance cortical function by reducing 'noise' and/or facilitating the processing of relevant signals suggests that the LC/noradrenergic system might act to enhance cognitive function under 'noisy' conditions where irrelevant stimuli impair performance. Experimental data from rodents, monkeys, and human patients support this prediction.

In rodents, depletion of forebrain NE generally does not impair performance of simple learning and memory tasks: these tests presumably place little demand on the cortex, and NE facilitatory mechanisms may be superfluous. An exception to this rule is the aged (Leslie et al. 1985; Collier et al. 1988) or in the combined LC/nucleus basalis lesioned animal (Haroutunian et al. 1990), suggesting that NE actions may become significant to simple learning in the compromised brain. However, NE depletion does produce deficits in the performance of young, otherwise intact animals on a variety of tasks when irrelevant stimuli are presented during testing. Thus, the addition of distracting visual stimuli at the choice point in a T-maze produces a much greater disruption of performance in NE depleted rats than in sham-treated animals (Roberts et al. 1975; Oke \& Adams, 1978). Similarly, the presentation of irrelevant, auditory stimuli interrupts the visual discrimination performance of rats with forebrain NE depletion, although these animals perform normally under nondistracting conditions (Carli et al. 1983). Further, NE depletion increases conditioned responses to irrelevant stimuli while decreasing responses to relevant stimuli (Lorden et al. 1980; Selden et al. 1990).

In primates, the prefrontal cortex (PFC) serves a critical role in inhibiting the processing of irrelevant stimuli (Knight et al. 1981; Woods \& Knight, 1986). In monkeys, this region can be functionally subdivided, with the dorsolateral PFC associated with proper performance in the delayed-response task, a test of spatial working memory. Monkeys with bilateral PFC lesions are markedly and permanently impaired on this task (Goldman-Rakic, 1987) and are especially vulnerable to interference from irrelevant stimuli (Bartus \& Levere, 1977).

Experimental evidence collected from non-human primates suggests an important, facilitatory role of NE in the proper regulation of PFC-dependent behaviour through actions at $\alpha_{2}$ noradrenergic receptors. Thus, noradrenergic $\alpha_{2}$-agonists, such as clonidine or guanfacine, improve performance in dorsolateral PFC-dependent tasks in monkeys with catecholamine (CA) depletion produced by MPTP (Schneider \& Kovelowski, 1990), reserpine (Cai et al. 1992), local infusion of 6-hydroxydopamine (6-OHDA) into the PFC (Arnsten \& Goldman-Rakic, 1985), or ageing (Arnsten \& Goldman-Rakic, 1985; Arnsten et al. 1988). Further, systemically-administered $\alpha_{2}-$ agonists lose their cognitive-enhancing effects when the PFC is ablated (Arnsten \& Goldman-Rakic, 1985), demonstrating that intact PFC circuitry is necessary for these drugs to have beneficial effects.

Aged monkeys are a useful model for studying NE effects on PFC function because they have naturally occurring catecholamine loss in the PFC (Goldman-Rakic \& Brown, 1981), and PFCdependent cognitive deficits. Thus, aged monkeys are more vulnerable to interference from irrelevant stimuli (Bartus, 1979) and show marked impairment on the delayed response task (Bartus, 1979). Administration of clonidine or guanfacine to aged monkeys markedly improves performance of the delayed response task (Arnsten et al. 1988), especially when irrelevant information is present during the delays (Jackson \& Buccafusco, 1991; Arnsten \& Contant, 1992). These beneficial effects of $\alpha_{2}$-agonists are independent of their ability to induce hypotension or sedation (Arnsten et al. 1988) and are blocked by $\alpha_{2}-$, but not $\alpha_{1}$-antagonists (Arnsten \& GoldmanRakic, 1985; Arnsten et al. 1988).

Pharmacological studies indicate that enhancement of PFC-related cognitive function results 
from stimulation of a subtype of $\alpha_{2}$-receptor with high affinity for guanfacine: the $\mathrm{Ri}$ site of Boyajian \& Leslie (1987), which appears to be the $\alpha_{2}$ A-site of Bylund (Uhlen \& Wikberg, 1991). The gene for this subtype is thought to reside on chromosome 10 (Bylund et al. 1992), and pharmacological analysis of the cloned receptor is consistent with a post-synaptic $\alpha_{2}$-receptor (Regan et al. 1988).

Behavioural pharmacological experiments also indicate that cognitive enhancement results from stimulation of post-synaptic $\alpha_{2}$-receptors. Therefore, as mentioned, clonidine has beneficial effects in monkeys with CA depletion, consistent with actions at post- rather than pre-synaptic receptors. Further, low ('pre-synaptic') doses of clonidine actually impair performance in aged monkeys (Arnsten et al. 1988), while higher, beneficial doses are blocked by the post-synaptic $\alpha_{2}$-antagonists, SKF104078 or SKF104856 (Arnsten \& Contant, 1992).

At least some of these beneficial effects of $\alpha_{2}$-agonists are due to drug actions directly in the PFC. In aged monkeys, infusion of clonidine into the PFC, but not nearby premotor cortex, improves delayed response performance (unpublished observations). Further, in young monkeys with 6OHDA lesions restricted to the PFC, clonidine's potency directly relates to the degree of NE depletion in the PFC, consistent with drug actions at supersensitive post-synaptic $\alpha_{2}$-receptors in this cortical region (Arnsten \& Goldman-Rakic, 1985).

NE stimulation of $\alpha_{2}$-receptors appears to be more important for PFC function than for abilities dependent on posterior cortical areas. Thus, $\alpha_{2}$-agonists produce marked improvement in aged and young CA-depleted monkeys performing PFC-dependent tasks such as delayed response (Arnsten et al. 1988) or delayed match-to-sample (Jackson \& Buccafusco, 1991), but have less influence on visual discrimination (Arnsten \& Goldman-Rakic, 1985) or delayed nonmatch-to-sample (Arnsten \& Goldman-Rakic, 1990) tasks. These latter tasks are thought to depend primarily on inferior and medial temporal lobe function, respectively.

\section{CLINICAL IMPLICATIONS}

One implication of the experimental observations described above concerns cognitive dysfunction associated with normal ageing. Humans display marked decrements in performance on PFCdependent tasks with ageing, similar to those observed in monkeys. For example, Davis et al. (1990) observed age-related impairments in performance on the Stroop Interference and Wisconsin Card Sort tests, even at relatively early stages in the ageing process. Thus, the ability of $\alpha_{2}$-agonists to improve performance of PFC-dependent tasks in aged monkeys suggests the possibility that these drugs may be of benefit in the treatment of cognitive decline associated with ageing in humans. However, this hypothesis remains to be tested.

That $\alpha_{2}$-agonists can exert beneficial effects on PFC-dependent abilities in humans has been documented in studies of patient populations with cognitive disorders not related to ageing. For example, clonidine improved PFC-dependent function in patients with Korsakoff's amnesia (Stroop Interference test, Word Fluency and Memory Recall) (Mair \& McEntee, 1986; Moffoot et al. 1992), schizophrenia (Trails B, memory recall (Fields et al. 1988)), and attention deficit disorder (attention regulation and impulsiveness) (Hunt et al. 1985). Extensive studies of Korsakoff's patients have shown that clonidine's ability to improve memory correlates with the degree of NE loss as indicated by CSF MHPG, consistent with an action of clonidine at postsynaptic $\alpha_{2}$-receptors (McEntee \& Mair, 1990). Most notably, recent SPECT imaging studies of Korsakoff's patients have revealed that clonidine's ability to improve word fluency is significantly correlated with increased regional cerebral blood flow in the PFC (Moffoot et al. 1993). These observations indicate that $\alpha_{2}$-agonists selectively enhance PFC function in human as well as nonhuman primates. Finally, analogous to monkeys, cognitive performance of humans with cortical lesions due to Alzheimer's disease is not improved by $\alpha_{2}$-agonists (Schlegel et al. 1989), demonstrating the need for intact PFC circuitry. 


\section{FUTURE DIRECTIONS}

To summarize, results from various investigations of LC/NE function reveal a surprising degree of cohesion: whether at the level of the single cell, populations of cells, or whole animal (behaviour), NE increases responses to relevant stimuli (signal) and/or facilitates suppression of responses to irrelevant stimuli (noise). Thus, an emerging view is one in which the LC/noradrenergic system exerts widespread effects at multiple levels of neuronal organization that facilitate cortical processing of sensory information and enhance cognitive processes that guide behaviour. At present, the evidence suggests that global changes in cortical efficiency may be mediated by $\alpha_{1}-$ and $\beta$-receptors, whereas more specific cortical actions (e.g. within PFC) may involve post-synaptic $\alpha_{2}-$ receptors. Additional studies are needed to address this hypothesis.

An implication of the hypothesis that optimal cognitive and behavioural performance is dependent on actions of the LC/noradrenergic system is that malfunction of this system might contribute to cognitive dysfunction associated with certain psychiatric and/or neurological disorders. A major impediment to the testing of this latter hypothesis is a lack of appropriate methodology for the specific manipulation of this system in humans. Although a pharmacological approach is desirable, there are a number of complicating issues associated with this approach.

First, most noradrenergic drugs exert peripheral as well as CNS effects. Many of the peripheral effects are either intolerable or could affect cognitive function through non-specific actions. Secondly, the recent identification of multiple subtypes of $\beta-, \alpha_{1}-$ and $\alpha_{2}$-receptors complicates matters, at least in the short term, in that the majority of currently available drugs do not display a high degree of selectivity for these recently identified subtypes.

Future studies will provide critical information concerning the multiplicity of noradrenergic receptor subtypes and structure-function relationships of these subtypes. This information is essential for the design of subtype-selective agonists and antagonists that exert minimal effects in the periphery. In turn, these drugs will provide the tools to improve the study of cognitive functions subserved by individual receptor subtypes. It is hoped that a clearer picture of the role(s) of individual receptors and the pharmacological tools to manipulate these receptors specifically will lead to the development of treatments targeted for specific cognitive dysfunction.

C. W. BERRIDGE, A. F. T. ARNSTEN AND S. L. FOOTE

\section{REFERENCES}

Arnsten, A. F. T. \& Contant, T. A. (1992). Alpha-2 adrenergic agonists decrease distractability in aged monkeys performing the delay response task. Psychopharmacology 108, 159-169.

Arnsten, A. F. T. \& Goldman-Rakic, P. S. (1985). Alpha-2 adrenergic mechanisms in prefrontal cortex associated with cognitive decline in aged nonhuman primates. Science 230, 1273-1276.

Arnsten, A. F. T. \& Goldman-Rakic, P. S. (1990). Analysis of alpha2 adrenergic agonist effects on the delayed nonmatch-to-sample performance of aged rhesus monkey. Neurobiology of Aging 11, 583-590.

Arnsten, A. F. T., Cai, J. X. \& Goldman-Rakic, P. S. (1988). The alpha-2 adrenergic agonist guanfacine improves memory in aged monkeys without sedative or hypotensive side effects. Journal of Neuroscience 8, 4287-4298.

Aston-Jones, G. \& Bloom, F. E. (1981). Activity of norepinephrinecontaining locus coeruleus neurons in behaving rats anticipates fluctuations in the sleep-waking cycle. Journal of Neuroscience 1 , 876-886.

Bartus, R. T. (1979). Effects of aging on visual memory, sensory processing and discrimination learning in a nonhuman primate. In Sensory Systems and Communication in the Elderly (Aging Vol. 10) (ed. J. M. Ordy and K. Brizzee), pp. 85-114. Raven Press: New York.
Bartus, R. T. \& Levere, T. E. (1977). Frontal decortication in thesus monkeys: a test of the interference hypothesis. Brain Research 119, 233-249.

Berridge, C. W. \& Foote, S. L. (1991). Effects of locus coeruleus activation on electroencephalographic activity in neocortex and hippocampus. Journal of Neuroscience 11, 3135-3145.

Berridge, C. W., Page, M., Valentino, R. J. \& Foote, S. L. (1991) Modulation of forebrain EEG by the locus coeruleusnoradrenergic (LC/NE) system. Society for Neuroscience Abstracts 17,1541

Berridge, C. W., Page, M., Valentino, R. J. \& Foote, S. L. (1993) Effects of locus coeruleus inactivation on forebrain electroencephalographic activity. Neuroscience (in the press).

Boyajian, C. L. \& Leslie, F. M. (1987). Pharmacological evidence for alpha-2 adrenoceptor heterogeneity: differential binding properties of $[3 \mathrm{H}]$ rauwolscine and $[3 \mathrm{H}]$ idazoxan in rat brain. Journal of Pharmacology and Experimental Therapeutics 241, 1092-1098.

Bylund, D. B., Blaxall, H. S., Iversen, L. J., Caron, M. G., Lefkowitz R. J. \& Lomasney, J. W. (1992). Pharmacological characteristics of $\alpha-2$ adrenergic receptors: comparison of pharmacologically defined subtypes with subtypes identified by molecular cloning Molecular Pharmacology 42, 1-5.

Cai, J. X., Ma, Y., Xu, L. \& Hu, X. (1992). Reserpine impairs spatial working memory performance in monkeys: reversal by the alpha2 adrenergic agonist clonidine. (Submitted.) 
Carli, M., Robbins, T. W., Evenden, J. L. \& Everitt, B. J. (1983) Effects of lesions to ascending noradrenergic neurones on performance of a 5-choice serial reaction task in rats; implications for theories of dorsal noradrenergic bundle function based on selective attention and arousal. Behavioural Brain Research 9 $361-380$.

Collier, T. J., Gash, D. M. \& Sladek, J. R. Jr. (1988). Transplantation of norepinephrine neurones into aged rats improves performance of a learned task. Brain Research 448, 77-87.

Davis, H. P., Cohen, S., Gandy, M., Colombo, P., VanDusseldorp, G., Simolke, N. \& Romano, J. (1990). Lexical priming deficits as a function of age. Behavioral Neuroscience 104, 288-297.

Dohlman, H. G., Thorner, J., Caron, M. G. \& Lefkowitz, R. J. (1991). Model systems for the study of seven-transmembrane segment receptors. Annual Review of Biochemistry 60, 653-688.

Fields, R. B., Van Kammen, D. P., Peters, J. L., Rosen, J., Van Kammen, W. B., Nugent, A., Stipetic, S. \& Linnoila, M. (1988). Clonidine improves memory function in schizophrenia independently from change in psychosis. Schizophrenia Research 1, 417-423.

Foote, S. L. \& Morrison, J. H. (1987). Extrathalamic modulation of neocortical function. Annual Review of Neuroscience 10, 67-95.

Foote, S. L., Freedman, F.E. \& Oliver, A. P. (1975). Effects of putative neurotransmitters on neuronal activity in monkey auditory cortex. Brain Research 86, 229-242.

Foote, S. L., Aston-Jones, G. \& Bloom, F. E. (1980). Impulse activity of locus coeruleus neurons in awake rats and monkeys is a function of sensory stimulation and arousal. Proceedings of the National Academy of Sciences, USA 77, 3033-3037.

Foote, S. L., Bloom, F. E. \& Aston-Jones, G. (1983). The nucleus locus coeruleus: new evidence of anatomical and physiological specificity. Physiological Reviews 63, 844-914.

Goldman-Rakic, P. S. (1987). Circuitry of the primate prefronta cortex and the regulation of behavior by representational memory In Handbook of Physiology, The Nervous System, Higher Functions of the Brain, Sect. 1, Vol. V, Pt. I (ed. F. Plum), pp. 373-417. American Physiological Society: Bethesda, MD.

Goldman-Rakic, P. S. \& Brown, R. M. (1981). Regional changes of monoamines in cerebral cortex and subcortical structures of aging rhesus monkeys. Neuroscience 6, 177-187.

Harley, C. W. \& Sara, S. J. (1992). Locus coeruleus burst induced by glutamate trigger delayed perforant path spike amplitude potentiation in the dentate gyrus. Experimental Brain Research 89 , $581-587$

Haroutunian, V., Kanof, P. D., Tsuboyama, G. \& Davis, K. L. (1990). Restoration of cholinomimetic activity by clonidine in cholinergic plus noradrenergic lesioned rats. Brain Research 507, 261-266.

Heginbotham, L. R. \& Dunwiddie, T. V. (1991). Long-term increase in the evoked population spike in the $\mathrm{CAl}$ region of rat hippocampus induced by $\beta$-adrenergic receptor activation. Journal of Neuroscience 11, 2519-2527.

Hobson, J. A., McCarley, R. W. \& Wyzinski, P. W. (1975). Sleep cycle oscillation: reciprocal discharge by two brainstem neuronal groups. Science 189, 55-58.

Hopkins, W. F. \& Johnston, D. (1984). Frequency-dependent noradrenergic modulation of long-term potentiation in the hippocampus. Science 226, 350-351.

Hopkins, W. F. \& Johnston, D. (1988). Noradrenergic enhancement of long-term potentiation at mossy fibre synapses in the hippocampus. Journal of Neurophysiology 59, 667-687.

Hunt, R. D., Minderaa, R. B. \& Cohen, D. J. (1985). Clonidine benefits children with Attention Deficit Disorder and Hyperactivity: report of a double-blind placebo-crossover therapeutic trial. Journal of the American Academy of Child Psychiatry 5, 617-629.

Jackson, W. J. \& Buccafusco, J. J. (1991). Clonidine enhances delayed matching-to-sample performance by young and aged monkeys. Pharmacology, Biochemistry, and Behavior 39, 79-84.

Jones, C. R. \& Palacios, J. M. (1991). Autoradiography of adreno- ceptors in rat and human brain: $\alpha$-adrenoceptor and idazoxan binding sites. Progress in Brain Research 88, 271-291.

Knight, R. T., Hillyard, S. A., Woods, D. L. \& Neville, H. J. (1981) The effects of frontal cortex lesions on event-related potentials during auditory selective attention. Electroencephalography and Clinical Neurophysiology 52, 571-582.

Leslie, F. M., Loughlin, S. E., Sternberg, D. B., McGaugh, J. L., Young, L. E. \& Zornetzer, S. F. (1985). Noradrenergic changes in senescent memory loss. Brain Research 359, 292-299.

Lorden, J. F., Rickett, E. J., Dawson, R. \& Pelleymounter, M. A. (1980). Forebrain norepinephrine and the selective processing of information. Brain Research 190, 569-573.

McCormick, D. A., Pape, H. C. \& Williamson, A. (1991). Actions of norepinephrine in the cerebral cortex and thalamus: implications for function of the central noradrenergic system. Progress in Brain Research 88, 293-305.

McEntee, W. J. \& Mair, R. G. (1990). The Korsakoff syndrome: a neurochemical perspective. Trends in Neuroscience 13, 340-344.

Mair, R. G. \& McEntee, W. J. (1986). Cognitive enhancement in Korsakoff's psychosis by clonidine: a comparison with $\mathrm{L}$-dopa and ephedrine. Psychopharmacology 88, 374-380.

Moffoot, A., O'Carroll, R. E., Murray, C., Dougall, N., Ebmeier, K. P. \& Goodwin, G. M. (1993). Effects of clonidine on cognition and rCBF in Korsakof's psychosis. Psychological Medicine (in the press).

Oke, A. F. \& Adams, R. N. (1978). Selective attention dysfunction in adult rats neonatally treated with 6-hydroxydopamine. Pharmacology, Biochemistry, and Behavior 9, 429-432.

Pineda, J. A., Foote, S. L. \& Neville, H. J. (1989). Effects of locus coeruleus lesions on auditory, long-latency, event related potentials in monkey. Journal of Neuroscience 9, 81-93.

Regan, J. W., Kobilka, T. S., Yang-Feng, T. L., Caron, M. G., Lefkowitz, R. J. \& Kobilka, B. K. (1988). Cloning and expression of a human kidney cDNA for an alpha-2 adrenergic receptor subtype. Proceedings of the National Academy of Sciences, USA 85, 6301-6305.

Roberts, D. S. C., Price, M. T. C. \& Fibiger, H. C. (1975). The dorsal tegmental noradrenergic projection: an analysis of its role in maze learning. Journal of Comparative Physiological Psychology 90, $363-372$

Schlegel, J., Mohr, E., Williams, J., Mann, U., Gearing, M. \& Chase, T. N. (1989). Guanfacine treatment of Alzheimer's disease. Clinical Neuropharmacology 12, 124-128.

Schneider, J. S. \& Kovelowski, C. J. (1990). Chronic exposure to low doses of MPTP. I. Cognitive deficits in motor asymptomatic monkeys. Brain Research 519, 122-128.

Selden, N. R. W., Robbins, T. W. \& Everitt, B. J. (1990). Enhanced behavioral conditioning to context and impaired behavioral and neuroendocrine responses to conditioned stimuli following ceruleocortical noradrenergic lesions: support for an additional hypothesis of central noradrenergic function. Journal of Neuroscience 10 , $531-539$.

Stanton, P. K. \& Sarvey, J. M. (1985). Depletion of norepinephrine, but not serotonin, reduces long-term potentiation in the dentate gyrus of rat hippocampal slices. Journal of Neuroscience $\mathbf{5}$, 2169-2176.

Stanton, P. K. \& Sarvey, J. M. (1987). Norepinephrine regulates long-term potentiation of both the population spike and dendritic EPSP in hippocampal dentate gyrus. Brain Research Bulletin 18 115-119.

Svensson, T. H., Bunney, B. S. \& Aghajanian, G. K. (1975) Inhibition of both noradrenergic and serotonergic neurons in brain by the alpha-adrenergic agonist clonidine. Brain Research 92 , 291-306.

Swick, D., Pineda, J. A., Holmes, T. C. \& Foote, S. L. (1988). Effects of clonidine on P300-like potentials in squirrel monkeys. Society for Neuroscience Abstracts 14, 1014.

Uhlen, S. \& Wikberg, J. E. S. (1991). Delineation of rat kidney alpha $2 \mathrm{~A}$ and alpha $2 \mathrm{~B}$-adrenoceptors with $[3 \mathrm{H}] \mathrm{R} \times 821002$ radioligand binding: computer modelling reveals that guanfacine is an alpha 
2A-selective compound. European Journal of Pharmacology 202, 235-243.

Vogt, B. A. (1991). The role of layer I in cortical function. In Cerebral Cortex, Vol. 9 (ed. E. G. Jones and A. Peters), pp. 49-80. Plenum Press: New York.

Woods, D. L. \& Knight, R. T. (1986). Electrophysiological evidence of increased distractibility after dorsolateral prefrontal lesions. Neurology 36, 212-216.

Woodward, D. J., Moises, H. C., Waterhouse, B. D., Hoffer, B. J. \& Freedman, R. (1979). Modulatory actions of norepinephrine in the central nervous system. Federation Proceedings 38, 2109-2116. 